\title{
Primary retroperitoneal perivascular epithelioid cell neoplasm: A case report
}

\author{
WENJIE LIANG ${ }^{1}$, CHANG XU $^{2}$ and FENG CHEN ${ }^{1}$ \\ ${ }^{1}$ Department of Radiology, The First Affiliated Hospital, College of Medicine, Zhejiang University, Hangzhou, \\ Zhejiang 310003; ${ }^{2}$ Department of Radiology, Hospital Affiliated to Binzhou Medical University, \\ Binzhou, Shandong 256603, P.R. China
}

Received August 12, 2014; Accepted April 27, 2015

DOI: $10.3892 / \mathrm{ol} .2015 .3210$

\begin{abstract}
Perivascular epithelioid cell neoplasm (PEComa) is a rare type of tumor, and primary retroperitoneal PEComa is rarer still. The present report discusses the case of a 51-year-old woman who was admitted to hospital following stiffness and discomfort in the upper right abdomen for longer than one month. Computed tomography identified a soft tissue mass in the rear right kidney, accompanied by clear cystic degeneration and bleeding. The borderline between the mass and the right kidney was not distinct. An enhanced scan revealed that the solid section of the mass was progressively enhanced. Following excision of the mass and the affected right kidney, the patient was pathologically diagnosed with retroperitoneal PEComa involving the right kidney. Short-term reexamination did not detect any recurrence.
\end{abstract}

\section{Introduction}

Perivascular epithelioid cell neoplasm (PEComa) is rare type of tumor that originates in mesenchymal tissues, and was first reported in 1996 (1). PEComa is defined by the World Health Organization Classification of Tumors of Soft Tissue and Bone as a mesenchymal tumor composed of perivascular epithelioid cells with unique histological properties and immunophenotypes (2). The PEComa family includes angiomyolipoma (AML), clear cell 'sugar' tumor (CCST), lymphangioleiomyomatosis (LAM), clear cell myomelanocytic tumor of the ligamentum teres/falciform ligament (CCMMT) and PEComa not otherwise specified (PEComa NOS) (3). PEComas rarely originate in the retroperitoneum and, even when this does occur, the majority of tumors are benign (4). Retroperitoneal PEComas generally occur in

Correspondence to: Dr Wenjie Liang, Department of Radiology, The First Affiliated Hospital, College of Medicine, Zhejiang University, 79 Qingchun Road, Hangzhou, Zhejiang 310003, P.R. China

E-mail: baduen.c@163.com

Key words: tumor, computed tomography, kidney women of $\sim 50$ years of age (4). Patients with retroperitoneal PEComa generally do not present with any discomfort, but occasionally exhibit non-specific symptoms, for example back pain, abdominal pain or a sense of oppression $(4,5)$.

The current study reports a rare case of primary retroperitoneal malignant PEComa involving the nearby retroperitoneal organs, which demonstrated similar imaging manifestations to those of a stromal tumor.

\section{Case report}

A 51-year-old female was admitted to hospital after experiencing stiffness and discomfort in the upper right abdomen for longer than one month, and subsequently, an opacity in the retroperitoneum was revealed by ultrasonography. The patient's symptoms of stiffness and discomfort were accompanied by a limitation in deep breathing and nighttime dyscoimesis of unknown cause. The stiffness recovered spontaneously after several hours. The patient did not present with any additional symptoms, for example nausea, vomiting, diarrhoea, melena, coughing or expectoration. During laboratory tests, routine urine analysis revealed the following: Erythrocytes, 27.2/ $\mu \mathrm{l}$ (normal, 0.0-22.7/ $\mu \mathrm{l}$ ); urobilinogen, $50 \mu \mathrm{mol} / \mathrm{l}$ (normal, 1.7-30.0 $\mu \mathrm{mol} / \mathrm{l}$ ) and bacteria, 425.6/ $\mu 1$ (normal, $0.0-130.7 / \mu 1$ ). Blood routine was normal, while levels of tumor markers, including $\alpha$-fetoprotein, carcinoembryonic antigen, carbohydrate antigens (19-9, 125 and 15-3) and ferritin, were also normal. Whole abdomen enhanced computed tomography $(\mathrm{CT})$ identified a massive soft-tissue density shadow of $11.1 \times 10.7 \times 20.0 \mathrm{~cm}$ to the rear of the right kidney. Multi-nodular and massive low-density shadows were observed in the mass, with striped high-density shadows within the low-density area (Fig. 1A). A plain scan revealed a CT value of 29.2 Hounsfield units (HU) in the solid section, while an enhanced scan demonstrated an unevenly enhanced solid section with a CT value of 44.6 $\mathrm{HU}$ at arterial phase and 77.1 $\mathrm{HU}$ at venous phase (Fig. 1B and C). The cystic section of the tumor was not enhanced. The tumor was enclosed and fed with an artery branching from the abdominal aorta and was clearly demarcated; however, the tumor borderline with the right kidney was unclear, and the right adrenal gland and kidney were displaced forward (Fig. 1D). The patient was preoperatively diagnosed as most likely having a retroperitoneal stromal tumor. Following 

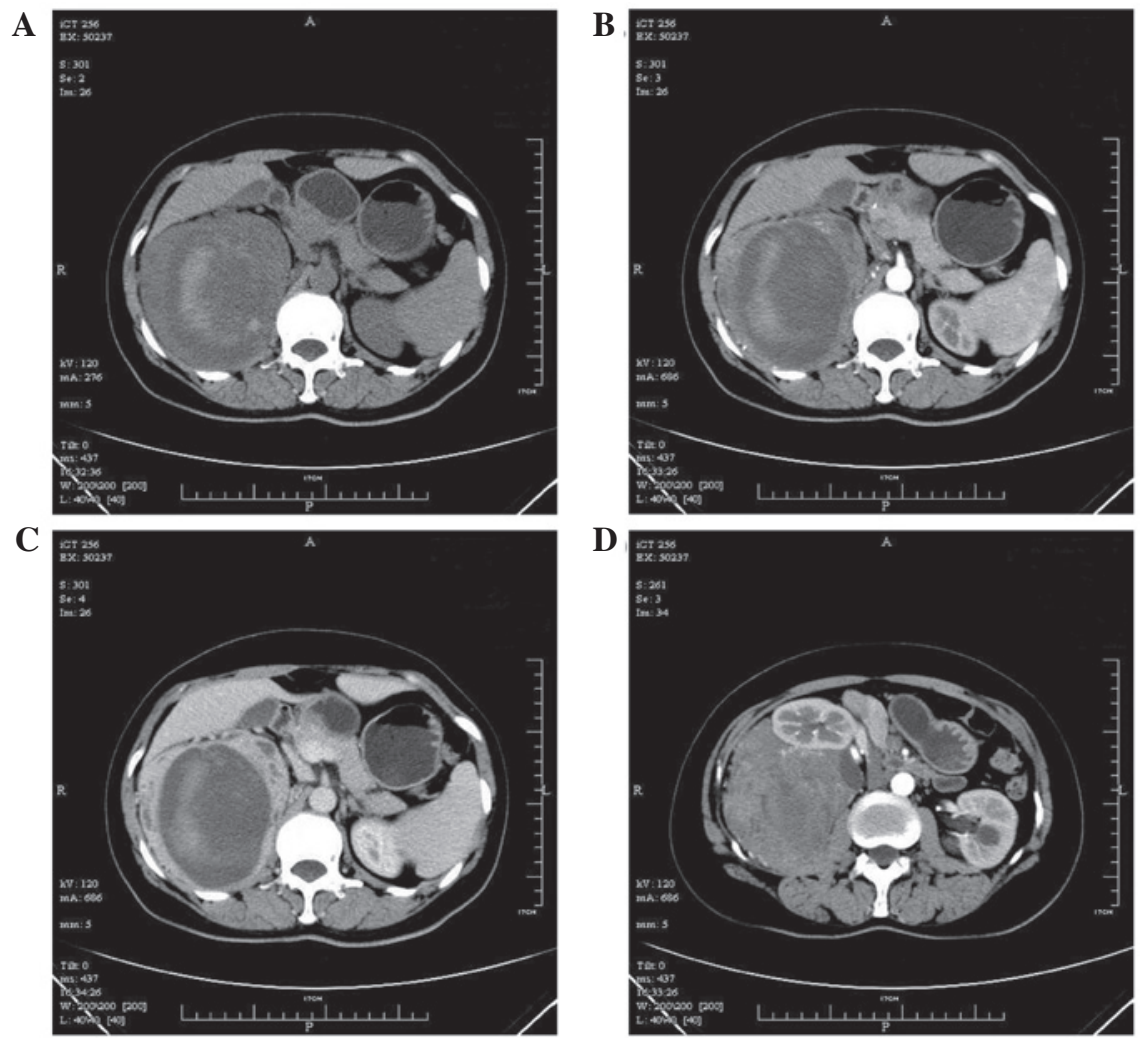

Figure 1. Enhanced computed tomography scan of the whole abdomen. (A) Transverse plane scan displays a massive soft-tissue mass occupying the rear of the right kidney with clear edges, but an unclear borderline with the right kidney, accompanied by cystic degeneration and bleeding. (B) Enhanced scan at arterial-phase demonstrated an unevenly enhanced tumor with multiple blood vessels. (C) Delayed phase, the solid section was progressively enhanced. (D) The fat gap between the right kidney and the mass disappeared.
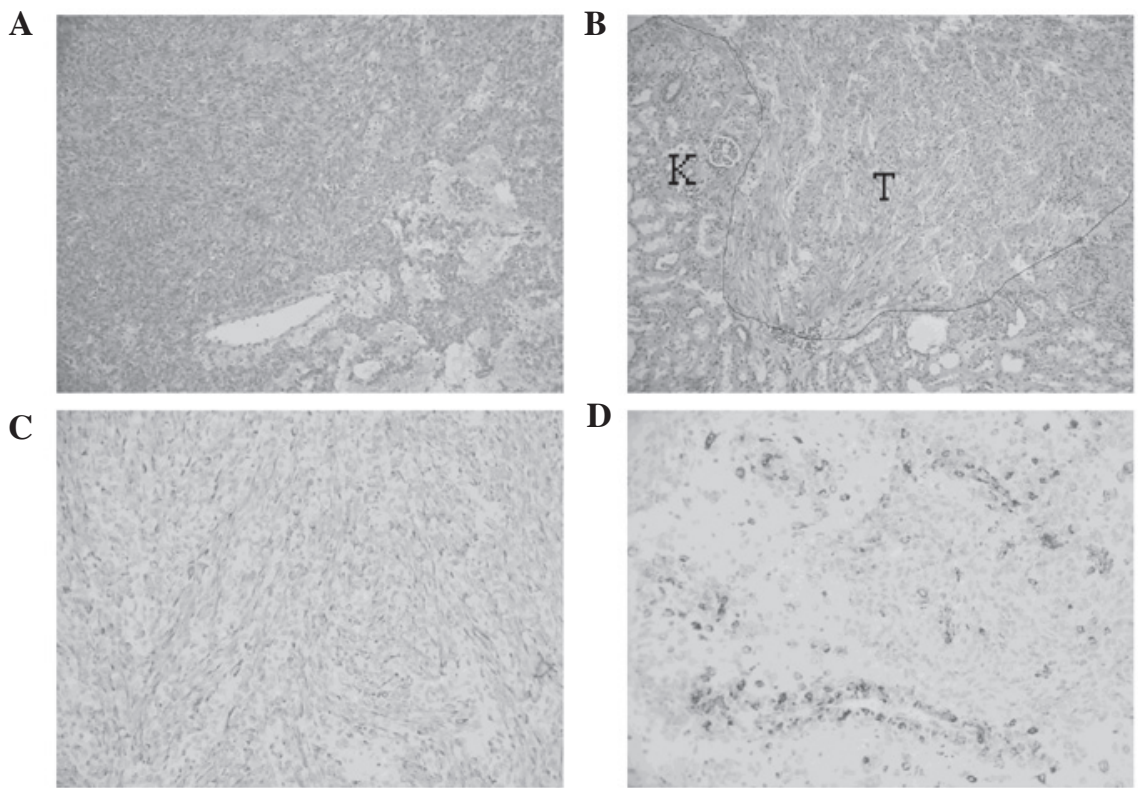

Figure 2. Pathological analysis. (A) Evenly-sized spindle cells around the vessels were arranged in bundles (hematoxylin and eosin staining; magnification, $\mathrm{x} 40$ ). (B) Histopathological slice analysis demonstrated renal parenchyma invasion by the tumor ( $\mathrm{T}$, tumor; $\mathrm{K}$, kidney; black line, roughly defined borderline between the tumor and kidney). (C and D) Immunohistochemical analysis revealed that tumor cells expressed (C) Desmin and (D) HMB-45 (magnification, x100).

complete preoperative preparation, the patient received resection of the retroperitoneal tumor and the involved right kidney. The findings observed during the surgery were as follows: A large mass $(12 \times 20 \mathrm{~cm})$ occupied the right side of the retroperitoneum with a clear margin and capsule. The upper pole of the tumor reached the diaphragmatic dome and was partially connected with the liver and the diaphragm muscle, while the lower pole remained dissociated, and its interior margin abutted the right kidney, which was also found to be partially involved during the separation. Pathological examination with 
hematoxylin and eosin staining revealed that the tumor cells were fusiform and arranged in bundles and microcapsules (Fig. 2A). The uniformly-sized cells occasionally demonstrated mitotic figures, and the focus invaded the kidney (Fig. 2B), but not the adrenal gland. Immunohistochemical (IHC) staining results were: Pan-cytokeratin(-), Melan-A(-), HMB-45(+), smooth muscle actin (SMA)(+), Desmin(+), CD34(-), P53(+) and Ki-67(+, 2\%) (Fig. 2C and D). The final diagnosis was retroperitoneal PEComa involving the kidney. The patient recovered well following surgery, without further treatment. No recurrence was identified during the follow up at 7 months post surgery.

Written informed consent for the use of medical data for teaching and research was obtained from the patient upon admission.

\section{Discussion}

PEComa is a rare type of tumor originating in mesenchymal tissues. The characteristics of PEComas-NOS are that, microscopically, the majority of perivascular epithelioid cells surround the blood vessels and are arranged radially around the lumen, forming bunch- and web-shaped structures (1-3). A PEComa is composed of two sections, the epithelioid cells surrounding the vessels and the spindle cells distal to the vessels, and the proportions of the two parts may vary significantly (3). Certain cases demonstrate clear nuclear atypia and mitosis (3). Sclerosing PEComa occurs when the tumor cells are embedded in the collagenized or hyalinized tumor stroma (4). A group of sclerosing PEComas were previously reported, and the majority occurred in the retroperitoneum (4). The case in the present study presented with typical manifestations of PEComa, but did not demonstrate obvious interstitial sclerosis. The typical IHC characteristics of PEComa are tumor cell expression of melanocyte and muscle cell markers; however, the expression levels of these markers differ between patients (4). The most sensitive markers are Desmin and HMB-45, followed by SMA, Caldesmon, and MiTF, while Melan-A and S-100 may be positively expressed in a lower proportion of cases (4). In the present case, HMB-45 was expressed positively and diffusely in the epithelioid cells, while SMA and Desmin were expressed diffusely in the spindle cells. Though most PEComas are benign, approximately half of PEComas-NOS are malignant $(5,6)$. As the number of reported cases is small, there are no consistent criteria for the diagnosis of benign or malignant PEComas. However, empirically-based criteria are defined as follows: Benign tumor, diameter $<5 \mathrm{~cm}$, no necrosis or vascular invasion, no infiltrative growth, with occasional nuclear atypia, and nuclear division $\leq 1 / 50 \mathrm{HPF}$, without definite malignant potential, with only nuclear pleomorphism/polykaryocytes or only tumor diameter $>5 \mathrm{~cm}$; malignant tumor, diameter $>5 \mathrm{~cm}$, necrosis, infiltrative growth or vascular invasion, clear nuclear atypia and nuclear division $\geq 1 / 50$ HPF (7). The present case exhibited occasional nuclear division, but the diameter of the lesion was $>5 \mathrm{~cm}$, and presented with bleeding, necrosis and invasion into the right kidney, all of which were consistent with the definition of malignant PEComa.

PEComas are associated with specific imaging manifestations according to their location. AMLs commonly occur in the liver or kidney, and may be diagnosed by the detection of fat components on $\mathrm{CT}$ or magnetic resonance (MR) imaging, with obvious enhancement on the enhanced scan. The key in diagnostic imaging is still the detection of fat components in low-fat AML or epithelioid AML (8-10). CCSTs are mainly benign and manifest as peripheral and smooth-edged quasi-circular nodules, without cavities or calcification, which may be markedly enhanced $(11,12)$. Malignant CCSTs are manifested as multi-nodules or masses, with occasional calcification and metastasis to the liver, adrenal gland or brain (13). LAM manifest on high-resolution CT as quasi-circular and almost wall-less cysts in diffuse distribution, likely accompanied by pneumothorax, pleural effusion or chylothorax, and occasionally with small AML and retroperitoneal lymphangioma in the kidneys (14). There has been little research regarding the imaging characteristics of CCMMT $(15,16)$. The imaging features of a group of malignant PEComas include signs of occurrence in the retroperitoneum, smooth-edged masses and uneven T2WI signals, likely with additional symptoms, including calcification, necrosis, bleeding, invasion into nearby veins and marked enhancement, however these imaging features are mostly non-specific (17). The major target organs of metastasis are the lungs and liver (17). The majority of reported PEComas occur in retroperitoneal organs. However, the case described in the present study originated in the retroperitoneum. Furthermore, it was initially reported that the PEComa invaded the nearby retroperitoneal organs (the right kidney), which may explain the increased erythrocyte content detected in the urine. In another 33 cases of PEComa occurring at various sites, two retroperitoneal cases displayed large lesions and enhancement, of which one was detected by fat components (16). Two recently reported primary retroperitoneal PEComas manifested as fat-rich masses with enhanced nodules or vascular structure $(5,18)$. Therefore, the detection of fat components and the display of blood-supply-rich components may be two valuable clues for imaging-based diagnosis of retroperitoneal PEComas. In the present case, the enhanced CT only clarified the rich-blood-supply soft tissues, and thus other rich-blood-supply lesions, for example sarcoma or stromal tumor, should be distinguished. No fat component was detected by plain CT scan or histopathological examination, however, marked intratumoral bleeding was detected, all of which differed from the results of previous imaging reports. It was hypothesized that the immaturity of tumor blood vessels is likely to induce ischemic necrosis and cystic degeneration in the focus, while the incomplete vascular walls may result in the rupture and bleeding of microvessels.

At present, primary and isolated metastatic PEComas are mainly treated by surgery, with good prognosis (3). Malignant PEComas may be treated with chemotherapy or immunotherapy, but with poor prognosis $(19,20)$. In the present case, the mass possessed clear edges, but had locally invaded the right kidney, and thus the excision of the tumor and associated right kidney was performed. Since the tumor was completely excised, no further treatment was applied. No recurrence was identified during the follow-up at 7 months post surgery.

In conclusion, retroperitoneal PEComa is a rare type of tumor, and its diagnosis remains difficult at preoperative imaging. However, diagnostic imaging will facilitate the selection of an appropriate treatment strategy. Locally invasive PEComa may be treated with surgery. The imaging characteristics and therapeutic methods for the diagnosis and treatment of retroperitoneal PEComa should be further evaluated. 


\section{References}

1. Zamboni C, Pea M, Martignoni G, et al: Clear cell 'sugar' tumor of the pancreas. A novel member of the family of lesions characterized by the presence of perivascular epithelioid cells. Am J Surg Pathol 20: 722-730, 1996.

2. Folpe AL: Neoplasms with perivascular epithelioid cell differentiation (PEComas). In: World Health Organization Classification of Tumors: Pathology and Genetics of Tumors of Soft Tissue and Bone. Fletcher CDM, Unni KK and Mertens F (eds). IARC Press, Lyon, pp221-222, 2002.

3. Armah HB and Parwani AV: Perivascular epithelioid cell tumor. Arch Pathol Lab Med 133: 648-654, 2009.

4. Hornick JL and Fletcher CD: Sclerosing PEComa: Clinicopathologic analysis of a distinctive variant with a predilection for the retroperitoneum. Am J Surg Pathol 32: 493-501, 2008.

5. Pata G, Tironi A, Solaini L, et al: Perivascular epithelioid cell tumor located retroperitoneally with pulmonary lymphangioleiomyomatosis: Report of a case. Surg Today 44: 572-576, 2014.

6. Alaggio R, Cecchetto G, Martignoni G, et al: Malignant perivascular epithelioid cell tumor in children: Description of a case and review of the literature. J Pediatr Surg 47: e31-e40, 2012.

7. Folpe AL, Mentzel T and Lehr HA, et a 1: Perivascular epithelioid cell neoplasms of soft tissue and gynecologic origin. Am J Surg Pathol 29: 1558-1575, 2005.

8. Zhao Y, Ouyang H, Wang X, et al: MRI manifestations of liver epithelioid and nonepithelioid angiomyolipoma. J Magn Reson Imaging 39: 1502-1508, 2014.

9. Kim JY, Kim JK, Kim N and Cho KS: CT histogram analysis: Differentiation of angiomyolipoma without visible fat from renal cell carcinoma at CT imaging. Radiology 246: 472-479, 2008.

10. Froemming AT, Boland J, Cheville J, et al: Renal epithelioid angiomyolipoma: Imaging characteristics in nine cases with radiologic-pathologic correlation and review of the literature. AJR Am J Roentgenol 200: 178-186, 2013.
11. Seo JB, Im JG, Seo JW and Yeon KM: Clear cell tumor of the lung. AJR Am J Roentgenol 166: 730-731, 1996.

12. Santana AN, Nunes FS, Ho N and Takagaki TY: A rare cause of hemoptysis: Benign sugar (clear) cell tumor of the lung. Eur J Cardiothorac Surg 25: 652-654, 2004.

13. Lim HJ, Lee HY, Han J, et al: Uncommon of the uncommon: Malignant perivascular epithelioid cell tumor of the lung. Korean J Radiol 14: 692-696, 2013.

14. Pallisa E, Sanz P, Roman A, et al: Lymphangioleiomyomatosis: Pulmonary and abdominal findings with pathologic correlation. Radiographics 22: 185-198, 2002.

15. Folpe AL, Goodman ZD, Ishak KG, et al: Clear cell myomelanocytic tumor of the falciform ligament/ligamentum teres: A novel member of the perivascularepithelioid clear cell family of tumors with a predilection for children and young adults. Am J Surg Pathol 24: 1239-1246, 2000.

16. Tan Y, Zhang H and Xiao EH: Perivascular epithelioid cell tumour: Dynamic CT, MRI and clinicopathological characteristics - analysis of 32 cases and review of the literature. Clin Radiol 68: 555-561, 2013.

17. Tirumani SH, Shinagare AB, Hargreaves J, et al: Imaging features of primary and metastatic malignant perivascular epithelioid cell tumors. AJR Am J Roentgenol 202: 252-258, 2014

18. Wildgruber M, Becker K, Feith M and Gaa J: Perivascular epitheloid cell tumor (PEComa) mimicking retroperitoneal liposarcoma. World J Surg Oncol 12: 3, 2014.

19. Jeon IS and Lee SM: Multimodal treatment using surgery, radiotherapy and chemotherapy in a patient with a perivascular epithelioid cell tumor of the uterus. J Pediatr Hematol Oncol 27: 681-684, 2005.

20. Parfitt JR, Bella AJ, Wehrli BM and Izawa JI: Primary PEComa of the bladder treated with primary excision and adjuvant interferon- $\alpha$ immunotherapy: A case report. BMC Urol 6: 20, 2006. 